\title{
Dissociating nonalcoholic steatohepatitis from hepatocellular carcinoma in obesity
}

\author{
Stergios A. Polyzos ${ }^{1}$, Jannis Kountouras ${ }^{2}$, Antonis Goulas ${ }^{1}$, Eleni Papakonstantinou ${ }^{1}$, \\ Paraskevi Papaioannidou ${ }^{1}$ \\ ${ }^{1}$ First Laboratory of Pharmacology, ${ }^{2}$ Second Medical Clinic, School of Medicine, Aristotle University of Thessaloniki, Thessaloniki, Greece \\ Correspondence to: Stergios A. Polyzos, MD, MSc, PhD. First Laboratory of Pharmacology, School of Medicine, Aristotle University of Thessaloniki, \\ Aristotle University campus, 54124 Thessaloniki, Greece. Email: spolyzos@auth.gr. \\ Comment on: Grohmann M, Wiede F, Dodd GT, et al. Obesity Drives STAT-1-Dependent NASH and STAT-3-Dependent HCC. Cell \\ 2018;175:1289-306.
}

Submitted Jul 19, 2019. Accepted for publication Jul 25, 2019.

doi: 10.21037/hbsn.2019.07.18

View this article at: http://dx.doi.org/10.21037/hbsn.2019.07.18

\section{Introduction}

The prevalence of obesity has increased over the last decades reaching epidemic proportions: $39 \%$ of adults were overweight or obese in 2016 worldwide, according to World Health Association (https://www.who.int/news-room/ fact-sheets/detail/obesity-and-overweight). Moreover, obesity is linked to metabolic syndrome (MetS) and related comorbidities, including nonalcoholic fatty liver disease (NAFLD), type 2 diabetes mellitus (T2DM), cardiovascular disease (CVD) and malignancies, thus resulting in higher mortality for obese individuals (1).

NAFLD is currently considered as the most predominant chronic liver disease worldwide (2). NAFLD starts from simple steatosis (SS) that may progressively advance to nonalcoholic steatohepatitis (NASH), cirrhosis and hepatocellular carcinoma (HCC) (3). Mortality is higher in NASH patients, owing to hepatic (i.e., cirrhosis and HCC) and extra-hepatic morbidity, including CVD and malignancies (4). Therefore, the estimated burden of NAFLD on healthcare cost and resource utilization is regarded as significant (5). Notably, despite its high prevalence, there is currently no approved treatment of NAFLD (6).

Obesity has been linked not only with the upward trend of NAFLD prevalence, but also with its more severe phenotypes, including HCC (7). By 2014, the contribution of NAFLD to HCC was $12 \%$ in a European population (8). Importantly, the prevalence of NAFLD-related HCC increased from $2.6 \%$ in $1995-1999$ to $19.5 \%$ in $2010-2014$, following a similar trend in overweight/obesity (from 34\% to $52 \%$, respectively) (8). NASH-related cirrhosis is not a prerequisite for HCC, since it may occur in non-cirrhotic liver (9). However, the molecular links mediating a potential dissociation between NASH/fibrosis and HCC have been poorly investigated.

\section{Obesity-driven STAT-1 and STAT-3 signaling}

Recently, Grohmann et al., based on a series of elegant in vivo experiments, proposed that obesity leads to the development of NASH/fibrosis and HCC through different molecular pathways emanating from a common origin, namely the activation of signal transducer and activator of transcription (STAT)-1 (NASH/ fibrosis) and STAT-3 (HCC) pathways, as a result of the oxidative inactivation of T-cell protein tyrosine phosphatase (TCPTP) and, possibly, other protein tyrosine phosphatases (PTPs) (10).

The same group had previously shown that PTPs are extensively oxidized in the liver of mice that develop SS after they are fed a high-fat diet (HFD) (11). Based on that observation, they initially examined whether the hepatic oxidation of PTPs in obese mice contributes to the development of NASH and HCC. They showed that C57BL/6 mice fed a HFD (promoting obesity, IR and SS, but not NASH) resulted in increased oxidation of PTPs, including PTP1B and TCPTP; more importantly, this effect was even more evident in mice fed a mixed cholinedeficient (CD)-HFD (that additionally promotes the 
progression of SS to NASH) (10).

Given that PTP1B and TCPTP are negative regulators of Janus kinase (JAK)/STAT signaling, Grohmann et al. investigated whether oxidation of PTP1B and TCPTP induces the phosphorylation/activation of STAT-1, STAT3 and STAT-5. They showed that STAT-1 and STAT-3, but not STAT-5, phosphorylation was increased in the livers of HFD-fed mice, with additional increment in those of CD-HFD-fed mice (10). Moreover, abolishing TCPTP expression in the hepatocytes of transgenic C57BL/6 mice (Alb-Cre;Ptpn $2^{f l f f}$ ), which do not normally develop $\mathrm{NASH}$, fibrosis or HCC, resulted in the recruitment and infiltration of T-cells in the liver and progression of SS to NASH, fibrosis and HCC (10). Next came the dissection of STAT-1 and STAT-3 roles in the development of NASH/ fibrosis and HCC, respectively. Grohmann et al. used TCPTP-deficient C57BL/6 mice with heterozygous loss of either STAT-1 (Alb-Cre;Ptpn $2^{f l f l} ;$ Stat $1^{f l /+}$ ) or STAT-3 $\left(\right.$ Alb-Cre;Ptpn $2^{f l f l} ;$ Stat $\left.3^{f l /+}\right)$ to show that, following exposure to HFD, attenuation of STAT-1 signaling led to prevention of T-cell recruitment and progression to NASH, but not of HCC. Inversely, attenuation of STAT-3 signaling prevented $\mathrm{HCC}$ without affecting progression to NASH and fibrosis (10). In addition, analysis of liver tissue gene expression by unbiased RNA sequencing verified by targeted real-time PCR, produced STAT-specific patterns of expression associated with each particular phenotype: genes associated with T-cell recruitment ( $\mathrm{Cxcl}-9$ ) and fibrosis (Acta2, Tgfb) were specifically dependent on STAT-1, whereas a gene linked to tumorigenicity ( $F g l 1)$ was specifically associated with STAT-3. Notably, acute response and inflammationrelated genes (Saa1, Crp, Ifng, Tnf) were associated with both STAT-1 and STAT-3 activation (10). Most importantly, the investigators showed that treatment of TCPTP-deficient mice with the chemical carcinogen diethylnitrosamine (DEN), which leads to the development of liver tumors in the absence of NASH/fibrosis, was accompanied by a STAT-3 signaling activation (10).

Finally, the authors validated some of their findings in humans. First, the oxidation of PTPs co-migrating with TCPTP was higher in obese with NAFLD activity score (NAS) 2-4 than in obese without steatosis (NAS $=0$ ). Second, the phosphorylation of STAT-1 and STAT-3 was increased in the livers of obese patients with NAFLD (NAS 2-4) compared with non-obese individuals. Furthermore, the expression of CXCL9 and FGL1 was progressively increased from obese individuals without steatosis to those with SS and even more to those with NASH (10).

\section{Closing remarks and future directions}

The study by Grohmann et al. is the first to provide robust evidence for the dissociation between NASH/fibrosis and HCC in obesity. This dissociation had been previously suspected from clinical studies, which had revealed the presence of HCC in non-cirrhotic NAFLD patients (9). The authors were based on the results of their previous work (11) and went forward by using a rational step-bystep investigative process and state-of-the-art methodology. Their results warrant observational clinical studies to validate their findings in obese individuals. If validated, then there are certain research and clinical implications. First, surveillance for HCC should be expanded to all obese patients with NAFLD, since HCC may occur without $\mathrm{NASH}$ or fibrosis. It is underlined that the surveillance for HCC is currently considered to be inadequate in NAFLD patients, a fact that delays HCC diagnosis and treatment (12). This is most likely due to the notion that NAFLD is generally a benign disease, which does not raise particular concern among health care providers and policy makers (7). Moreover, effective screening is hampered by limited knowledge of the pathways underlying the pathogenesis of HCC in NAFLD and a lack of tools needed to stratify the associated risk (12). In this regard, the Grohmann et al. study provides fertile ground for studies investigating the degree of oxidative inactivation of TCPTP or other effectors and mediators of STAT-3 signaling as diagnostic tools for the early diagnosis of HCC in obese individuals with NAFLD. Another possibility is the design of diagnostic tests exploiting the reported close association of CCXL9 expression with early events in the progression of NAFLD to NASH, and, most importantly, that of FGL1 expression to HCC. Moreover, compounds that help prevent or minimize oxidation of PTPs involved in STAT1 and STAT-3 activation or directly inhibit STAT-3 need to enter the drug discovery/development pipeline to provide drugs that may help prevent HCC in NAFLD patients. In fact, TTI-101, an oral inhibitor of STAT-3, is at a phase I clinical trial in patients with various types of malignancy ( $\mathrm{n}=30)$, including HCC (NCT03195699).

Yet another dimension of the findings reported by Grohmann et al. is the possible involvement of cytokines, adipokines and other mediators, acting via STAT1 and STAT-3 pathways, in NASH/fibrosis and HCC development. For example, leptin, which is upregulated in obesity, was previously proposed to participate in the pathogenesis of NAFLD through a JAK/STAT-3 pathway $(13,14)$. However, it remains to be shown whether leptin 
upregulation contributes to the increased risk of $\mathrm{HCC}$ via the activation of STAT-3 signaling.

Although the Grohmann et al. study elucidated potential mechanisms promoting $\mathrm{NASH} /$ fibrosis and HCC, both these diseases are multifactorial, thus more than one pathogenetic mechanisms are involved in their pathogenesis. Therefore, STAT-1 and STAT-3 activation may separately promote NASH/fibrosis and HCC, respectively, but they are not expected to be the unique pathways in their pathogenesis. Thus, Grohmann et al. study provide evidence for HCC possibly arising without NASH/fibrosis, but it could not explain the higher rates of HCC observed in patients with more advanced NAFLD (9). Although NASH/fibrosis may not be prerequisites for HCC, still advanced fibrosis remains a strong risk factor of HCC (9). Indeed, at this point, it is hard to figure out how TCPTP inactivation could differentially affect STAT-1 and STAT-3 pathways, although one can imagine that PTPs with particular STAT-1 or STAT-3 specificity could also be involved. Also, one cannot exclude the possibility of crosstalk between different STAT pathways in the clinical setting.

In conclusion, the work by Grohmann et al. has opened a new range of possibilities that will keep NAFLD investigators busy for some time to come. The decoding of molecular pathways linking obesity, NAFLD and HCC is expected to reveal useful targets for the development of new diagnostic tools and medications for the specific treatment of NASH or HCC. Additionally, it has provided further molecular evidence for a major take home message: global efforts should mainly focus on the prevention of obesity (15); the interception of obesity epidemic may not only diminish its impact on the increasing rates of NASH and HCC, but will certainly have multiple-system health benefits.

\section{Acknowledgments}

None.

\section{Footnote}

Conflicts of Interest: The authors have no conflicts of interest to declare.

Ethical Statement: The authors are accountable for all aspects of the work in ensuring that questions related to the accuracy or integrity of any part of the work are appropriately investigated and resolved.

\section{References}

1. Global BMI Mortality Collaboration, Di Angelantonio E, Bhupathiraju ShN, et al. Body-mass index and allcause mortality: individual-participant-data meta-analysis of 239 prospective studies in four continents. Lancet 2016;388:776-86.

2. Younossi ZM, Golabi P, de Avila L, et al. The Global Epidemiology of NAFLD and NASH in Patients with type 2 diabetes: A Systematic Review and Meta-analysis. J Hepatol 2019. doi:10.1016/j.jhep.2019.06.021.

3. Polyzos SA, Kountouras J, Mantzoros CS. Adipose tissue, obesity and non-alcoholic fatty liver disease. Minerva Endocrinol 2017;42:92-108.

4. Rinella ME. Nonalcoholic fatty liver disease: a systematic review. JAMA 2015;313:2263-73.

5. Yoo ER, Ahmed A, Kim D. Economic burden and healthcare utilization in nonalcoholic fatty liver disease. Hepatobiliary Surg Nutr 2019;8:181-3.

6. Thanapirom K, Tsochatzis EA. Non-alcoholic fatty liver disease (NAFLD) and the quest for effective treatments. Hepatobiliary Surg Nutr 2019;8:77-9.

7. Polyzos SA, Kountouras J, Mantzoros CS. Obesity and nonalcoholic fatty liver disease: From pathophysiology to therapeutics. Metabolism 2019;92:82-97.

8. Pais R, Fartoux L, Goumard C, et al. Temporal trends, clinical patterns and outcomes of NAFLD-related HCC in patients undergoing liver resection over a 20-year period. Aliment Pharmacol Ther 2017;46:856-63.

9. Goh GB, McCullough AJ. Natural History of Nonalcoholic Fatty Liver Disease. Dig Dis Sci 2016;61:1226-33.

10. Grohmann M, Wiede F, Dodd GT, et al. Obesity Drives STAT-1-Dependent NASH and STAT-3-Dependent HCC. Cell 2018;175:1289-306.

11. Gurzov EN, Tran M, Fernandez-Rojo MA, et al. Hepatic oxidative stress promotes insulin-STAT-5 signaling and obesity by inactivating protein tyrosine phosphatase N2 . Cell Metab 2014;20:85-102.

12. Younes R, Bugianesi E. Should we undertake surveillance for HCC in patients with NAFLD? J Hepatol 2018;68:326-34.

13. Polyzos SA, Kountouras J, Zavos C, et al. The Potential Adverse Role of Leptin Resistance in Nonalcoholic Fatty Liver Disease: A Hypothesis Based on Critical Review of Literature. J Clin Gastroenterol 2011;45:50-4.

14. Polyzos SA, Kountouras J, Mantzoros CS. Leptin in nonalcoholic fatty liver disease: A narrative review. 
Metabolism 2015;64:60-78.

15. Boutari C, Polyzos SA, Mantzoros CS. Of mice and men: Why progress in the pharmacological management of

Cite this article as: Polyzos SA, Kountouras J, Goulas A, Papakonstantinou E, Papaioannidou P. Dissociating nonalcoholic steatohepatitis from hepatocellular carcinoma in obesity. Hepatobiliary Surg Nutr 2020;9(1):73-76. doi: 10.21037/ hbsn.2019.07.18 obesity is slower than anticipated and what could be done about it? Metabolism 2019;96:vi-xi. 